\title{
OCCURRENCE OF “BETA BURSTS" IN HUMAN FRONTAL CORTEX RELATED TO PSYCHOLOGICAL PARAMETERS
}

\author{
A. Kamp, C. F. M. Schrijer and W. Storm van Leeuwen \\ Institute of Medical Physics T.N.O., Utrecht (The Netherlands)
}

(Accepted for publication: February 18, 1972)

In the EEG recorded from the human scalp several activities have been discerned which, because of certain characteristic properties, have been set apart and named "specific activities". Obvious examples are alpha rhythms, mu rhythms and lambda waves but many other specific activities occurring in normal subjects or in patients are well known. In human subjects, with rare exceptions, the "specific activities" can be recorded only by means of scalp electrodes. From animal studies, however, it is known that they occur also in structures well out of range of scalp electrodes. Various of such activities have been described by many different investigators, of which will be mentioned as examples only the hippocampal theta rhythms related to alertness (Green and Arduini 1954) and the amygdalar $10-20 \mathrm{c} / \mathrm{sec}$ activities related to sniffing (Storm van Leeuwen et al. 1963, 1968). In man such activities can be studied only if diagnosis or therapy of cerebral afflictions necessitates the use of chronically indwelling electrodes in the brain. We have had the opportunity to study, in a psychiatric patient with such electrodes, a specific activity which we have not encountered in the literature and which, therefore, is presented here.

\section{Patient}

The patient was a 32-year-old woman with obsessional neurosis of such severity that no form of psychiatric or psychopharmacological therapy produced satisfactory relief of her complaints.

After extensive discussion with Dr. H. J. Crow (Burden Neurological Institute, Bristol, England) it was decided to carry out multifocal destruction of cingulate and orbital structures by means' of electrocoagulation with chronically implanted electrodes. It is not the place here to discuss the therapeutic value of the method. It may suffice to mention that in the course of 6 months of psychotherapy combined with multifocal coagulation remarkable relief of complaints and amelioration of the patient's state were obtained.

\section{METHODS}

The coagulation was carried out by means of electrodes implanted in the brain, as described by Crow et al. (1961). The electrodes consisted of strands of gold wire $(150 \mu)$, insulated except for $4 \mathrm{~mm}$ at the tip. On each side of the brain 16 electrodes were placed in orbital and inferior frontal structures and 18 electrodes in cingulate structures, making a total of 68 electrodes. The electrodes were also used for EEG recording by means of conventional 16-channel EEG apparatus (Offner $\mathrm{R}$ type and Elema Schönander) and by means of 16-channel radiotelemetry (Storm van Leeuwen and Kamp 1969). The electrical signals were stored by multichannel tape-recorders (24-track EMI and 14track Ampex). Various forms of signal analysis were performed on-line or subsequently off-line, from tape. In total 108 EEGs were recorded from this patient.

Most of the recording sessions, with the patient lying on a couch in a quiet, dimly lit room, differed from the usual EEG routine condition in that often a contingent negative variation (CNV) paradigm was presented. In many cases, however, she was investigated under 
special conditions or she was asked to perform other tasks. In these conditions usually 16-channel EEG radiotelemetry was carried out.

The conditions and the tasks which are of interest for the present investigation will be described separately in the section Resulis.

\section{RESULTS}

Of the many electrical phenomena recorded from the various structures, one was particularly noticeable since it arose occasionally conspicuously from background activity (Fig. 1) and since its occurrence was observed to be related to certain behavioural or psychological aspects. The phenomenon consisted of rhythmic paroxysmal activity at frequencies between approximately 16 and $28 \mathrm{c} / \mathrm{sec}$ : "beta bursts". These beta bursts had amplitudes between 100 and $200 \mu \mathrm{V}$, sometimes up to $400 \mu \mathrm{V}$ and durations of $0.3-1$ sec. The activity was encountered in various derivations from the left and right inferior frontal cortices. On the basis of X-ray measurements the relevant electrode positions were estimated to be in a transverse section $33-38 \mathrm{~mm}$ anterior to a vertical zero plane through the meati auditorii, $21-27 \mathrm{~mm}$ lateral from the midline (from atlas of Delmas and Pertuiset 1959). The activities of the two sides did not always occur simultaneously or with precisely the same frequencies. It should be mentioned that the electrodes on the two sides were not placed entirely symmetrically. The beta bursts on the left side were the more conspicuous and the following relates almost exclusively to these bursts.

The behavioural situations of the patient when beta bursts arose could be divided in two groups: (1) no obvious relation between the patient's behaviour and the occurrence of beta bursts and (2) obvious provocation of beta bursts by certain behavioural situations.

In the first group were: (a) the usual EEG recording situation, the patient reclining on a couch with eyes open or closed; (b) looking at projected slides of general interest : towns, landscapes, etc.; (c) walking around; (d) performing various activities such as writing, drawing, etc.; (e) general conversation with others (Fig. 1):

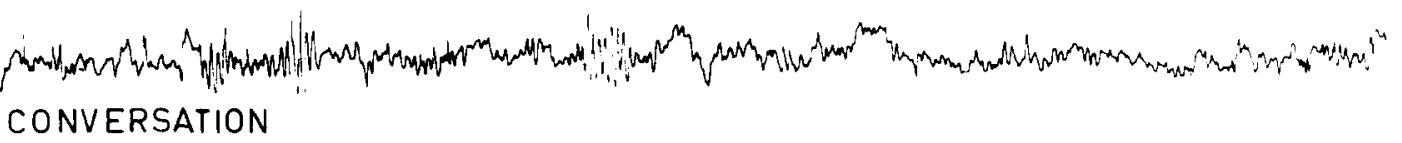

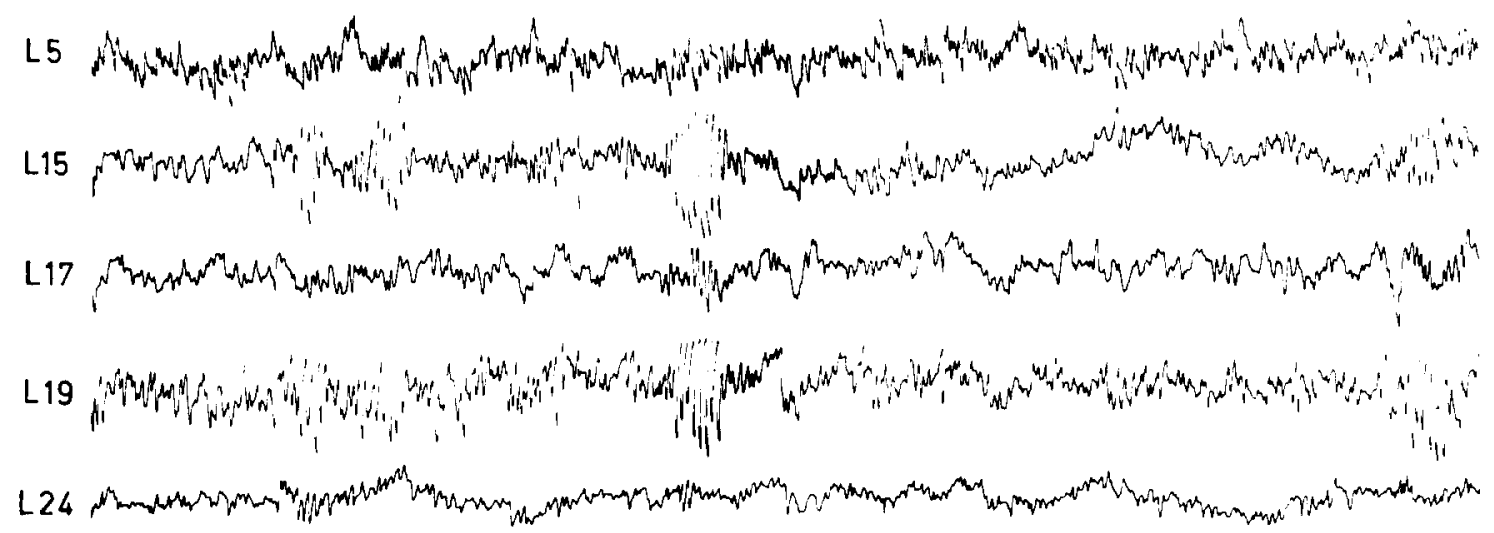

$\begin{array}{lll}\text { S.J. } 4-3-71 & 100 \mu \mathrm{V} \\ \text { EEG } 42 & \text { T. }=0,3 \mathrm{sec}\end{array}$

Fig. 1. Occurrence of paroxysms at frequencies between 16 and $26 \mathrm{c} / \mathrm{sec}-$ - beta bursts-in derivations from electrodes implanted in the left inferior frontal cortex (L5, L15, L17 and L19) while the patient is engaged in conversation (derivations against common relatively indifferent reference electrode in white matter). 


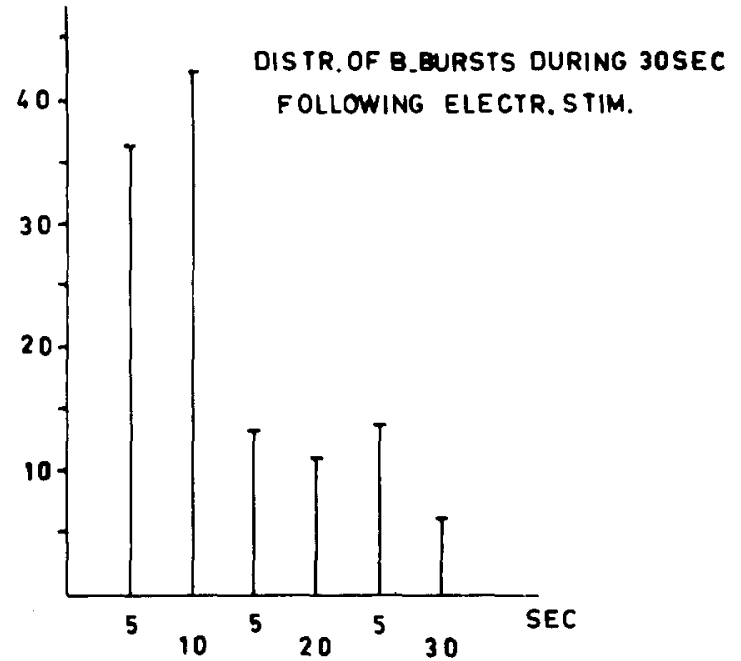

Fig. 2. Histogram of time of occurrence of beta bursts (in seconds) after electrical stimulation through implanted electrodes. The number of beta bursts is largest $5-10 \mathrm{sec}$ after stimulation. doctors, EEG technicians, etc. and (f) the usual interview with the psychiatrist.

Behavioural situations provocative of beta bursts were:

1. The periods following electrical stimulation, at different intensities, through each of the electrodes - a routine procedure for evaluating electrode positions in grey or white matterwhen the patient was asked: "Did you experience something?". The question each time was asked approximately $5-10 \mathrm{sec}$ after the end of stimulation and roughly at this time (questions and answers were not monitored) beta bursts occurred significantly more often than at other times (Fig. 2). There was no relation between occurrence of beta bursts and location of stimulated electrode.

2. Word association tests. During these tests more beta bursts occurred than at other times. The increase is demonstrated by comparing the 40 bursts occurring in a $10 \mathrm{~min}$ period during

\section{COM. REF. R 24}

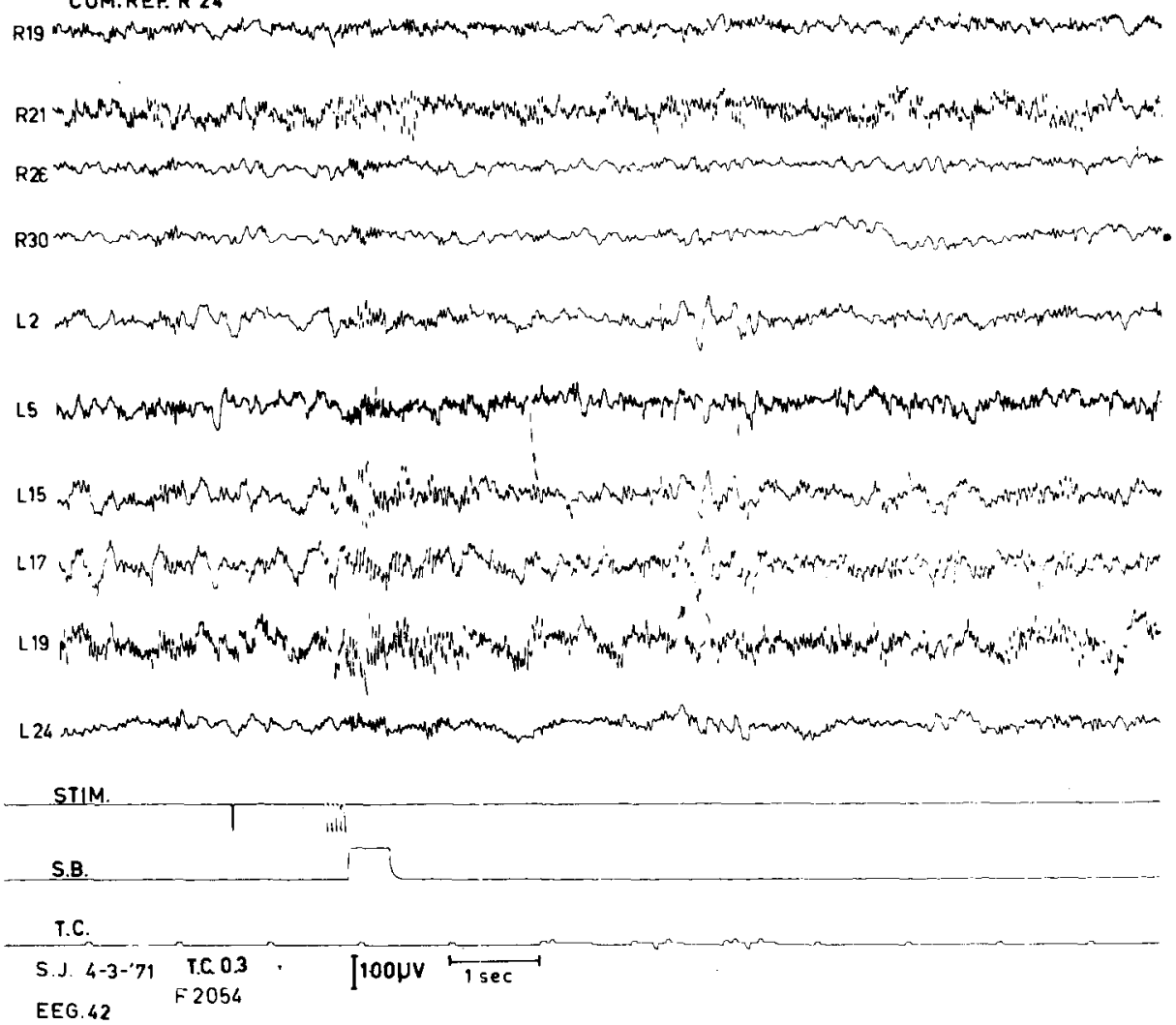

Fig. 3. Occurrence of beta bursts in inferior frontal cortex, left (L15, L17 and L19) and right (R21), during CNV paradigm (stim.; 1: click, 2: flashes; S.B. : switch button) during "normal" CNV (derivations to common reference as in Fig. 1). 
word association testing with the 11 bursts occurring in an equal period during the usual psychiatric interview. During the word association test the intervals between the presentation of the word and the patient's answer varied considerably: from a few seconds up to $2 \mathrm{~min}$. In the period of $10 \mathrm{~min} 46$ words were presented; with 30 of these one or more beta bursts occurred 29 of the answers were preceded immediately (within $1 \mathrm{sec}$ ) or accompanied by a beta burst.

3. The period of a $C N V$ paradigm. The paradigm was a classical one (Walter et al. 1964): warning click (or flash), followed after $1 \mathrm{sec}$ by a series of flashes (or clicks) the duration of which the patient could shorten by pressing a switch button at the right time. The occurrence of the beta bursts in relation to the paradigm varied somewhat in the course of many sessions, at each of which 40-70 CNV paradigms were presented. Chronologically the beta bursts arose in the following manner.
During the first $C N V$ sessions the beta bursts often occurred immediately after the patient's pressing of the button, significantly more often than at other periods (Fig. 3). In later sessions, when the CNV had been recorded a great many times, the beta bursts occurred less regularly and with smaller amplitudes. Their appearance tended to be associated with pressings which were either too early or considerably too late.

During equivocation experiments the amplitude of the beta bursts increased. The equivocation consisted in omitting randomly $(50 \%)$ the repetitive flashing, irrespective of the patient's button pressing. In Fig. 4 an example is shown of the occurrence of a beta burst slightly more than $1 \mathrm{sec}$ after the warning click, when no flashes were presented and while the patient did not press the button. To evaluate quantitatively the effect of equivocation on the amplitude of beta bursts, frequency analysis was carried out. by means of analogue bandpass filters, in the

COM. REF. R24

$R 19$.

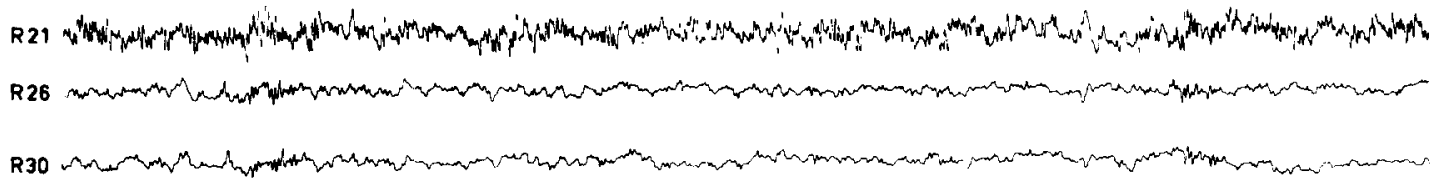

L2
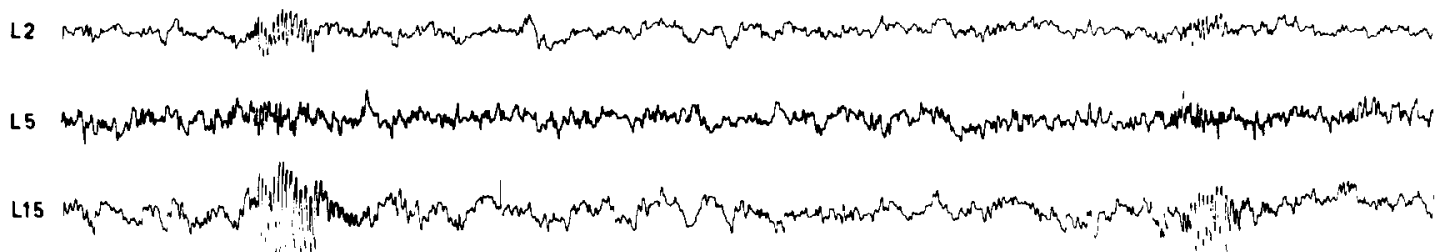

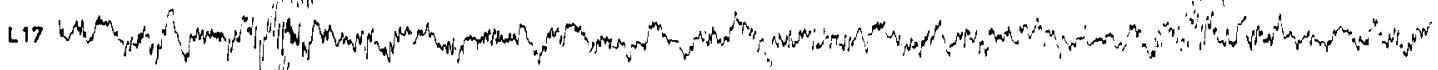

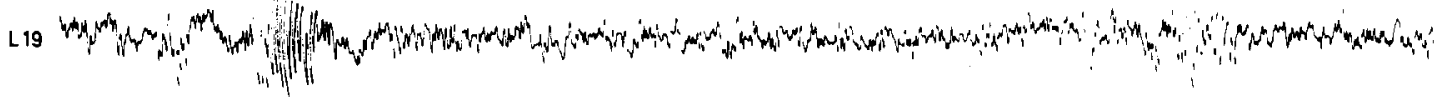

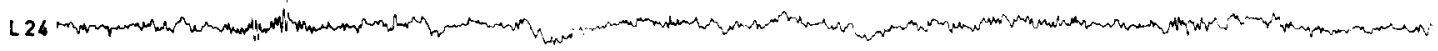
ST. SB

\begin{tabular}{|c|c|c|}
\hline T.C. & & \\
\hline S.J. 4.3.271 & T. C. $=0,3 \mathrm{sec}$ & $1 \mathrm{sec}$ \\
\hline EEG. 42 & $100 \mu v$ & \\
\hline F. 2057 & & \\
\hline
\end{tabular}

Fig. 4. Occurrence of beta bursts during equivocation. The burst arises at the time the flashes would have occurred (derivations as in Fig. 3). 

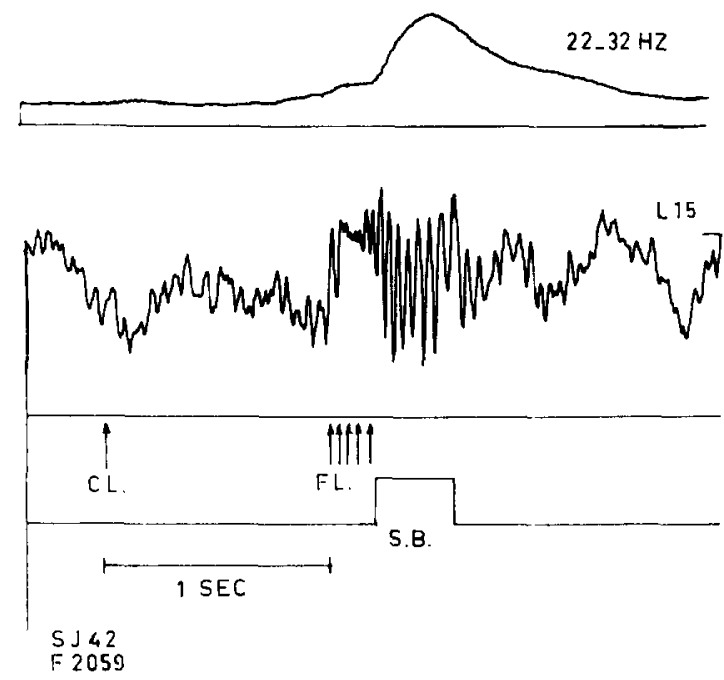

Fig. 5. Occurrence of beta burst in left inferior frontal cortex (L15) during "normal" CNV and, above, rectified output of $22-32 \mathrm{c} / \mathrm{sec}$ filter.

bands between $14-20$ and $22-32 \mathrm{c} / \mathrm{sec}$, over periods lasting $4 \mathrm{sec}$, starting $0.3 \mathrm{sec}$ before the warning click. The outputs of the bandpass filters were rectified and smoothed by means of low pass filters with time constants of $0.25 \mathrm{sec}$, and were stored in an average response computer (Mnemotron CAT $400 \mathrm{~B}$ ); numbers of comparable presentations were averaged.

In Fig. 5 the output of a $22-32 \mathrm{c} / \mathrm{sec}$ bandpass filter during a single presentation is given in the upper trace and, in Fig. 6, band $c$, averages over 22 presentations. As can be seen the beta bursts occurred during equivocation experiments when no flashes were presented; the latencies at which the bursts occurred in relation to the warning click in these latter cases did not appear to differ much from those during flashes (see also Table I).

To obtain objective and quantifiable data on the occurrence of the beta bursts in various conditions the outputs of the $22-32 \mathrm{c} / \mathrm{sec}$ filters were used. The peak amplitudes during the beta bursts were measured as well as the level of beta activity in the period preceding it (Fig. 6, $a$. values $Q$ and $P$ ). The quotient of these two values $\mathrm{Q} / \mathrm{P}$ was calculated and, when it concerned averages, normalized for the number of additions. Values of Q/P significantly larger than 1 indicate a significant increase of beta activity, values of 1 or less indicate no change or decrease of beta activity.

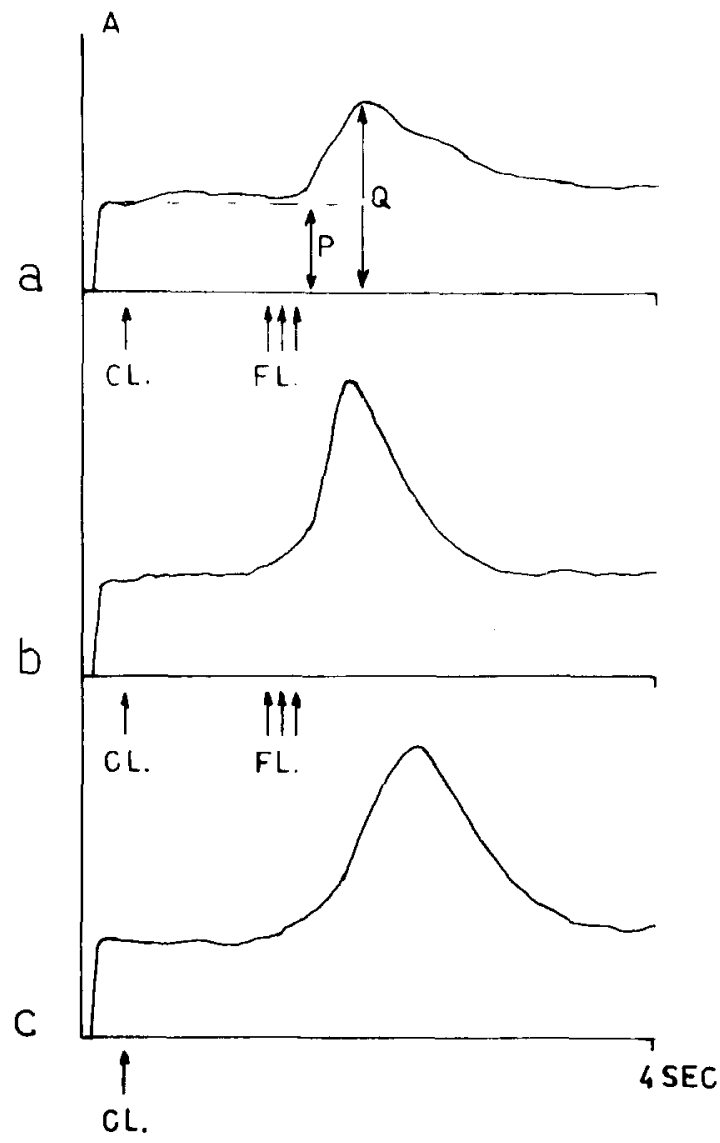

FR. BAND $22-32 \mathrm{HZ}, \mathrm{N}=22$

\section{S.J.38, 24.2.71 F 2052}

Fig. 6. Output of $22-32 \mathrm{c} / \mathrm{sec}$ during "normal" CNV $(a$ and $b)$ and during equivocation (c). $a: 1$ presentation; $b$ and $c$ : averages of 22 presentations. Amplitudes normalized for number of presentations. $\mathrm{P}$ and $\mathrm{Q}$ indicate the values for calculating the quotient $\mathrm{Q} / \mathrm{P}$.

TABLE I

\begin{tabular}{lllr} 
& $\mathrm{T}_{\mathrm{lo}}$ & $\mathrm{SD}$ & $\mathrm{N}$ \\
\hline CNV normal & 1.26 & 0.06 & 15 \\
CNV equiv. + & 1.24 & 0.09 & 5 \\
CNV equiv. & 1.22 & 0.05 & 5
\end{tabular}

$\mathrm{T}_{10}=$ Latency in seconds between click and onset of beta bursts. $\mathrm{N}=$ Number of averaged $22-32 \mathrm{c} / \mathrm{sec}$ filter outputs.

In Fig. 7, $a$ diagrams are presented of $\mathrm{Q} / \mathrm{P}$ values obtained at a number of consecutive experimental sessions (35th-42nd sessions) during the usual CNV paradigm, called "normal"

Electroenceph. clin. Neurophysiol., 1972, 33: 257-267 


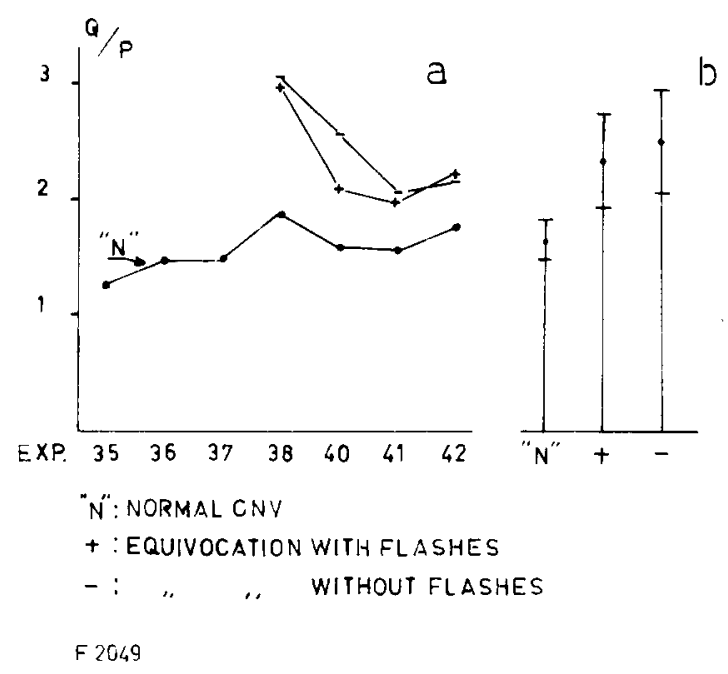

Fig. 7. Q/P values of averaged $22-32 \mathrm{c} / \mathrm{sec}$ filter outputs $(N=20)$ obtained during normal $C N V$ ("N") and during equivocation; + , presentations with flashes; - , presentations without flashes. In $a$ values are given from a number of recording sessions (exp. 35-42). Note considerable increase during equivocation, particularly during the first (session 38) In $b$ averages and standard deviations of the values in $a$

or " $\mathrm{N}$ " $\mathrm{CNV}$, and during equivocation. In the latter series half of the presentations are with flashes "+" and half without "-". The means and standard deviations of the values (Fig. 7,b) obtained during the normal CNV paradigm and during equivocation show that the values in the latter case are significantly larger than in the former, whereas the differences between equivocation with flashes and without flashes are not significant. From Fig. 7, $a$ it appears also that the increase of amplitude during consecutive equivocation experiments tends to diminish.

Extinction and reinforcement experiments showed a marked increase of beta burst amplitude (not shown in figure) during the first 5-10 presentations and a subsequent gradual decrease of the amplitude.

\section{Prediction during equivocation}

From conversations with the patient it appeared that during the equivocation series she tried mentally to predict whether or not flashes would occur, notwithstanding the fact that she had been told that the order was random. Consequently the patient was asked to indicate her prediction by saying "yes" or "no" after the warning clicks. To facilitate her doing so, the period between the click and the onset of the flashes was increased to $2 \mathrm{sec}$. Since flashing was omitted randomly in $50^{\circ}$, of the cases, the patient's prediction was right as often as wrong. It should be remarked here that, particularly in the later series of equivocation experiments, the patient pressed the button only after the onset of the flashes, apparently using the first flash as a warning signal.

The equivocation experiments were carried out in 4 series of experiments during which the patient was asked: (a) to predict, to indicate her prediction by saying "yes" or "no" and to press the button as usual (pred-ind-press); (b) to predirt. tc indicate ner prediction by saying "yes" or "no" but not to press the button, whether or not flashes occurred (pred-ind-no press); (c) to predict but not to indicate the prediction by saying "yes" or "no" and not to press (pred-no ind-no press); (d) not to predict, not to say "yes" or "no" and not to press, thus remaining entirely passive (no pred no ind-no press).

During the first three series of experiments beta bursts were observed whenever the patient predicted, with or without indicating her prediction and with or without pressing. These findings are presented in the diagram of Fig. 8. From this diagram it appears that there was a considerable increase of amplitude in the beta band on all occasions involving prediction and that the values were largest when flashes were presented, even when the patient did not indicate her prediction and did not press the button. A smaller increase was noticeable when the patient was instructed to remain passive, not predicting, not indicating and not pressing. Still, in this case, the values were larger with than without flashes.

4. Binary choice test. To investigate whether beta bursts were also provoked by other situations involving prediction, a test by means of a binary choice generator was carried out. The patient was presented with high and low tones in random order, which could be suppressed by pressing a switch. She was instructed to press a right hand switch at the high and a left hand switch at the low tones. Three series of experiments were performed in which the patient was asked whenever she had pressed a switch: (a) to predict whether the next tone would be high or 

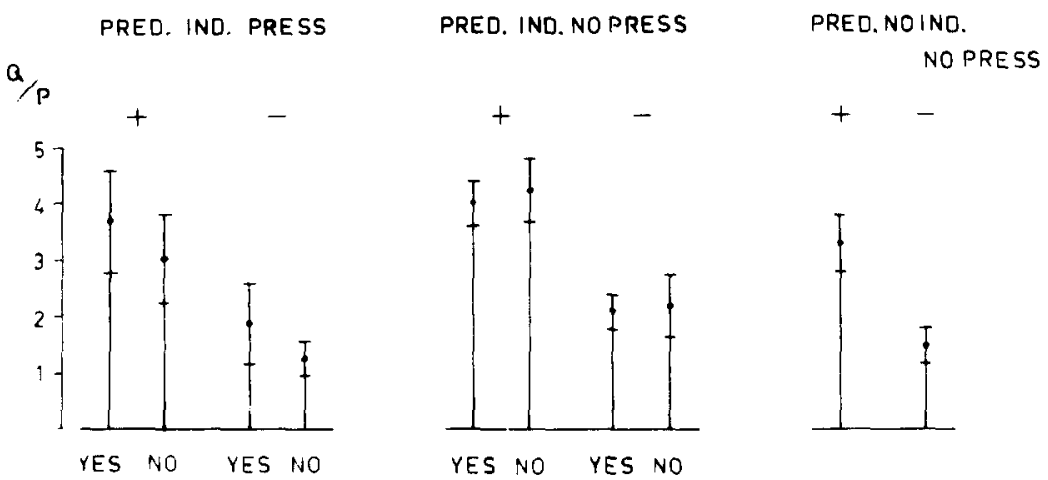
NO PRED.NO IND.
NO PRESS

F 2050

Fig. 8. Means and standard deviations of the Q/P values calculated over 25 or more presentations during the CNV paradigm with equivocation. All values larger than ! indicate increase of beta activity. Pred = prediction; ind = patient indicated prediction by saying "yes" or "no"; + =flashes occur; $-=$ flashes do not occur; press = patient is asked to press switch button.

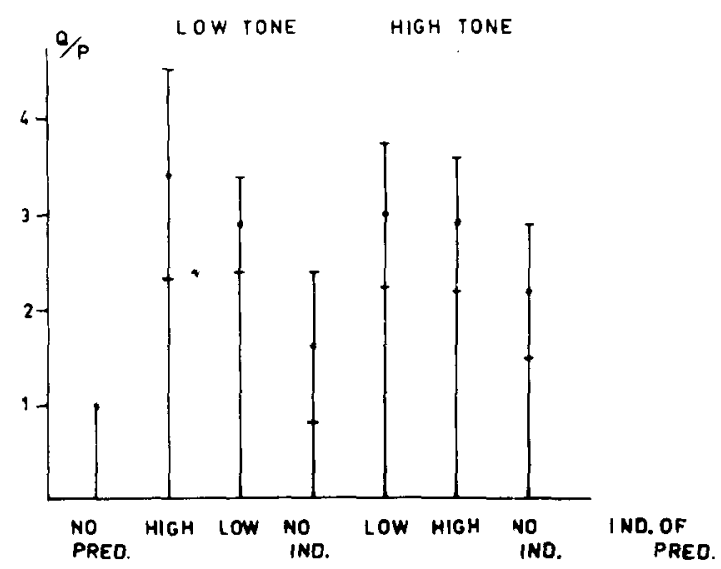

Fig. 9. Means and standard deviations of $Q / P$ values during binary choice test. Below $\mathrm{X}$ axis is indicated whether patient predicted "high" or "low" tone $(\mathrm{N}=>1 \mathrm{i})$.

low and to indicate her prediction by saying "high" or "low"; (b) to predict but not to indicate her prediction: (c) not to predict and, obviously, not to indicate.

As in the previous situations the peak amplitudes in the beta band were calculated in relation to background activity and diagrams of the values are shown in Fig. 9. From this figure it appears that there was considerable increase of beta activity in all situations involving prediction, whereas during non-prediction the values did not rise significantly above background level.

\section{Latency of beta bursts}

During the presentations of the CNV paradigms a certain time relation was observed between the warning click and the initiation of the beta bursts. To evaluate this relationship the time was measured between the click and the onset of rise of the $22-32 \mathrm{c} / \mathrm{sec}$ bandpass filter output, averaged over at least 12 presentations. Means and starfdard deviations were calculated of the values obtained from a number of averages (Table I). The latency between click and initiation of beta burst was slightly more than $1.2 \mathrm{sec}$

In addition, the latencies to initiation as well as to peak amplitude of beta bursts were measured and means and standard deviations were calculated as above during CNV experiments with prediction (Table II). In these cases the time between click and flash was increased to $2 \mathrm{sec}$. From these values it appears that the mean latency between click and initiation of beta bursts varied between 1.60 and $1,90 \mathrm{sec}$ and the latency of the peak amplitudes between 2.64 and $2.95 \mathrm{sec}$.

TABLE II

CNV with prediction and indication but no switch pressing.

$\begin{array}{cccccccc} & \mathrm{T}_{10} & \mathrm{SD} & \mathrm{T}_{\text {lr }} & \mathrm{SD} & \mathrm{T}_{1 \mathrm{p}} & \mathrm{SD} & \mathrm{N} \\ \mathrm{Yes}+ & 1.76 & 0.09 & 2.16 & 0.09 & 2.64 & 0.08 & 56 \\ \mathrm{No}+ & 1.90 & 0.10 & 2.21 & 0.03 & 2.69 & 0.02 & 45 \\ \text { Yes }- & 1.60 & 0.21 & & & 2.84 & 0.19 & 30 \\ \text { No }- & 1.80 & 0.17 & & & 2.95 & 0.29 & 55\end{array}$

$\mathrm{T}_{\mathrm{bo}}=$ Latency in seconds between click and onset of beta buists. $T_{1 \mathrm{r}}=$ Latency of sharp rise of amplitude. $\mathbf{T}_{1 \mathrm{p}}=$ Latency of peak amplitude. $\mathrm{SD}=$ standard deviation. $\mathrm{N}=$ total number of samples distributed over averages. 


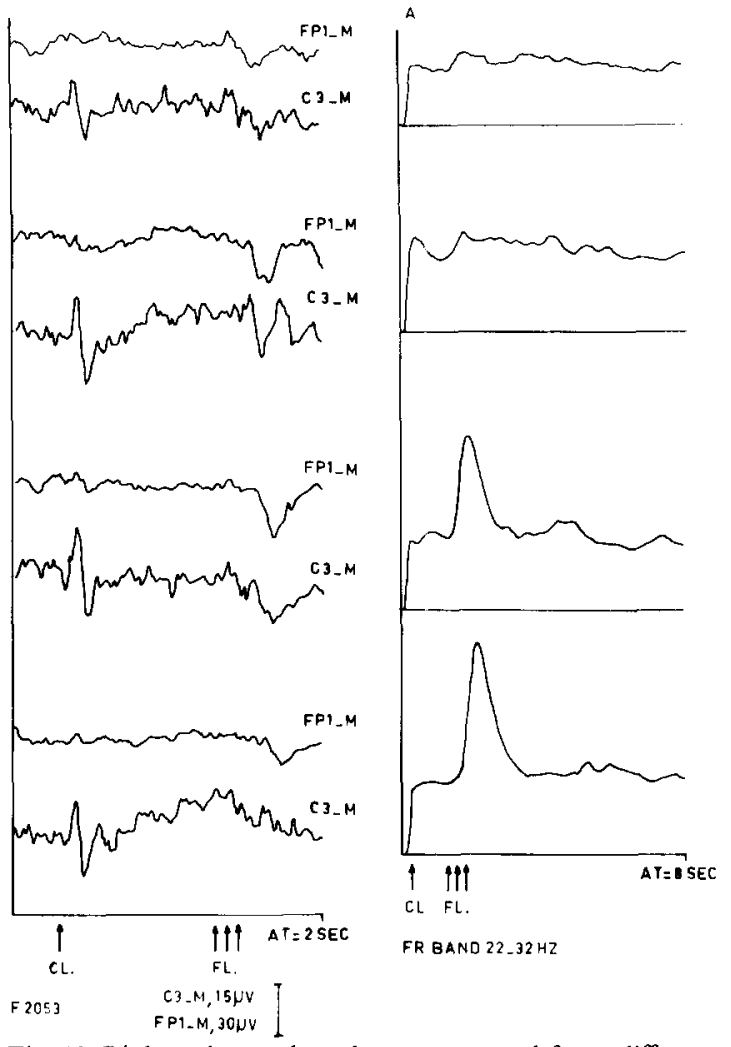

Fig. 10. Right column : beta bursts averaged from different presentations showing either small or no beta bursts in the primary trace (1st and 2nd averages) or large beta bursts (3rd and 4 th averages). $A=$ relative amplitude. Left column: eye movement $\left(\mathrm{FP}_{\mathrm{I}}-\mathrm{M}\right)$ and $\mathrm{CNV}(\mathrm{C} 3-\mathrm{M})$ recorded and averaged simultaneously with beta bursts in right column.
Shape of $C N V$ and beta bursts

To obtain some insight into this question, in the primary trace groups of $12 \mathrm{CNV}$ paradigms were selected which were accompanied by clear and large beta bursts, and also groups of 12 with small or no beta bursts. Then, as described above, average continuous frequency analyses were performed of the groups with and the groups without beta bursts and the CNVs pertaining to these groups were averaged. Comparison of the averaged beta activity and the simultaneously averaged CNVs showed (Fig. 10) that some of the large beta bursts were accompanied by a clear CNV and others by a small or absent CNV. It was concluded that no obvious relation existed between occurrence of beta bursts and wave form of $\mathrm{CNV}$.

\section{Heart rate and beta bursts}

It was observed that during the CNV paradigm a small decrease of heart rate occurred approximately $1 \mathrm{sec}$ after the warning click and during the beta bursts. To evaluate this change the time between heart beats was measured in two periods before, one period during and one period after the beta bursts. The period consisted of the intervals between 3 consecutive heart beats (see Fig. 11). To circumvent the variations of average heart rate during the various presentations, the difference between the first two periods $(A-B)$ was compared with the differences between the second and third $(B-C)$ and the

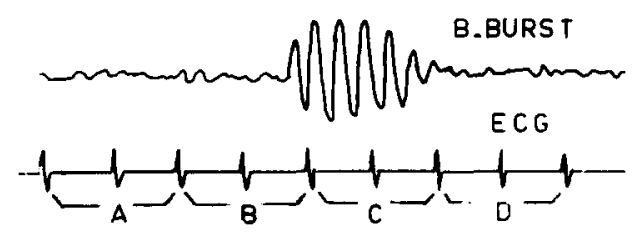

\begin{tabular}{l|cccc|ccc|cccccc|}
\multicolumn{1}{c}{$\bar{D}$} \\
\cline { 2 - 11 }
\end{tabular}

Fig. 11. Relation between beta burst and heart rate during "normal" CNV, CNV with equivocation and during "spontaneous" beta bursts. Values are measured in millimeters between three consecutive heartbeats; two periods before occurrence of beta bursts $(A$ and $B)$, one period during $(C)$ and one after $(D) . \overline{\mathrm{D}}=$ mean differences; $\sigma \mathrm{D}=$ standard deviations and $\mu \mathrm{d}=$ confidence limits $(P=0.05)$. 
third and fourth $(C-D)$. Of the differences the mean $(Q)$, standard deviation $(\sigma D)$ and confidence limits $(\mu \mathrm{d})(P=0.05)$ were calculated. When the upper and lower confidence limits both had negative or positive values a significant decrease or increase of heart rate occurred. These values were determined at the time that beta bursts occurred during normal CNVs, during equivocation and when beta bursts occurred "spontaneously". The data clearly indicated significant heart rate slowing of $5-10 \%$ during beta bursts in each of these situations. The decrease of heart rate was smallest during normal CNVs.

\section{Beta bursts in scalp EEG}

In a number of recordings unsuccessful attempts were made to observe beta bursts in the scalp EEG whenever they occurred in the depth derivations. In these experiments the patient was slightly restless and the scalp EEG was somewhat disturbed by muscle artefacts. No cross-correlations have been performed as yet between the activities recorded from depth and from surface.

\section{DISCUSSION}

As appears from the results, the beta bursts in the inferior frontal cortex occurred at a certain phase of stimulus-performance paradigms, for example the CNV, but they appeared also occasionally when the patient was not reacting to intentionally delivered stimuli. In fact the bursts were observed first during these latter "free" conditions. Our interest in intrinsic rhythmic brain activities--intensified by our observations in dogs with chronically implanted electrodesled to further study of the bursts: possible relations to behaviour or to other electrical brain activities, etc. In relation to the latter the question arose whether the beta bursts in the inferior frontal cortex might be regarded as a "specific activity". Considering the symmetric and restricted localization, frequency, form and reactivity this question can be answered in the affirmative.

The occurrence of the beta bursts at a certain phase of the CNV paradigm raises the question whether it has specific relations to this phenomenon. This possibility is unlikely because the beta bursts were provoked also by other paradigms, for example: (1) when asking the patient "Did you experience anything?" after electrical stimulation, and (2) during word association tests. In addition, no obvious relation was observed between occurrence of beta bursts and wave form of the CNV. Moreover, the relation of beta bursts to the CNV paradigm did not seem to be rigid. In fact quantitative measurements and the calculation of mean values and standard deviations were necessary to prove the significance of the relation.

Beta bursts were obviously provoked during conditions involving prediction, be it the CNV paradigm or other. However, since the bursts occurred after the moment that the patient could check the outcome of the prediction, they need not be related to it as such. Furthermore, beta bursts were also provoked during behavioural situations which did not involve prediction.

Since the patient had no preceding information to give her grounds for "prediction", the question has been raised (W. Grey Walter) whether logically it ought not to be called "guessing". From an objective point of view this certainly is correct. Subjectively, however, from the patient's point of view it undoubtedly was "prediction" and thus we have adhered to this latter term.

To obtain some insight into the behavioural aspects which are associated with the occurrence of beta bursts the situations during which they were enhanced may be compared. Commonly they were well known by the patient. From experience or instruction she knew beforehand precisely how the stimulus-performance paradigms were enacted. Another common aspect was that the beta bursts were accompanied by a small $\left(5-10^{\circ}{ }_{0}\right)$ but significant decrease of heart rate. These facts have led us to the hypothesis that in the periods preceding the beta bursts a certain "mental tension" is built up and that the occurrence of the beta bursts is associated with a sudden brief release of this "tension" immediately after the patient has checked the outcome of a stimulus-response paradigm, whether CNV or otherwise. Obviously, similar sudden brief release of "tension" may take place also during mental processes inaccessible to the experimenter, for example during thinking. Such processes might 
account for the occurrence of the "spontaneous" beta bursts accompanied by decreased heart ratc. In this context it should be mentioned that slowing of heart rate in a certain phase of the CNV paradigm was described first by Lacey and Lacey (1971). who found it in normal subjects and in patients.

A discrepancy appears to exist between the amplitudes of beta bursts in early CNV equivocation experiments without prediction and the later ones with prediction. In the latter the amplitudes of the beta bursts were significantly larger when flashes were presented than when they were omitted (Fig. 8), whereas in the former they were not (Fig. 7). An explanation for this may be obtained by studying the latency of the bursts. In the early series without prediction the latency showed very little variation (Table I), indicating that the patient was well trained and was able to estimate accurately the 1 sec interval between click and flashes. Thus, the non-occurrence of flashes was estimated almost as precisely as the occurrence. In the later series, with prediction. the time between click and flashes was increased to $2 \mathrm{sec}$ and the latencies were much more variable, indicating decreased precision of the patient's time estimation. This was particularly noticeable during non-occurrence of flashes. From Table II it appears that the onset of the beta bursts slightly precede (by approximately $0.2 \mathrm{sec}$ ) the initiation time for the flashes, whether they were actually presented or not. When flashes were presented the latencies to peak amplitudes were shorter $\left(\mathrm{T}_{1 n}: 2.64\right.$ and 2.69$)$ and the standard deviations smaller (sd: 0.08 and 0.02 ) than when no flashes were presented $\left(T_{1 \mathrm{p}}: 2.84\right.$ and 2.95 ; sd: $(0.19$ and 0.29$)$. This indicates that the occurrence of the flashes led to increased steepness of rise of beta bursts, and to increased precision of the moment at which the patient could check the outcome of her prediction. In terms of our hypothesis this would indicate that the outcome of the prediction is easier and more precisely checked when flashes appear than when they do not.

Finally, it is necessary to discuss the question whether beta bursts as described above occur in all persons, normal or diseased, or are a peculiarity of this one psychiatric patient in whom we have encountered them. Until many more patients have been investigated this question cannot be answered unambiguously. For the time being we offer the hypothesis that the phenomenon is indeed a "normal" specific activity. but we are well aware that this is supported only by a few indications and not by adequate proof. The indications are:

1. The beta bursts were recorded in the inferior frontal cortices of both hemispheres. They obviously do not represent a unilateral focal disturbance.

2. The beta bursts were recorded over the entire recording period of 8 months, in at least 70 of the 108 recorded EEGs (not all included derivations from the inferior frontal cortex). Over this period the frequency, amplitude, location and reactivity essentially remained unchanged. In the beginning of the recording period no coagulations had been performed and thus the occurrence of the beta bursts cannot be attributed to the multifocal lesions.

3. Similar beta bursts have been observed in psychiatric patients with implanted electrodes by the Bristol group (McCallum 1971, personal communication), but in their cases quantitative values were not determined and the relation of beta burst occurrence to the CNV paradigm was suspected but not proved.

4. The Lacey's (1971) observation that the decrease of heart rate occurs during the $\mathrm{CNV}$ paradigm in all subjects, normal and patient. similar to the decrease of heart rate in our patient related to the occurrence of beta bursts.

Obviously, so long as beta bursts cannot bo recorded from scalp electrodes it will not be possible to prove unambiguously that they constitute a normal phenomenon.

In conclusion, thereforc, for the present it is proposed that the beta bursts occurring in inferior frontal cortex are a specific electrical brain activity related to brief and sudden release of mental tension, such as can occur during checking the outcome of predictions made in various stimulus performance paradigms.

\section{SUMMARY}

1. In a psychiatric patient with electrodes implanted in various brain structures the occurrence of paroxysmal 16-26 c/sec activity --.. "beta 
bursts" -in the inferior frontal cortex is described.

2. The beta bursts occurred during "free" behavioural situations but could be provoked also by various stimulus-performance paradigms, such as those of the contingent negative variation $(\mathrm{CNV})$, the binary choice etc.

3. No significant relation was observed between occurrence of beta bursts and wave form of the CNV.

4. A significant increase of beta bursts was observed during situations involving prediction by the patient.

5. A significant small decrease of heart rate was observed during occurrence of beta bursts.

6 . The hypothesis is proposed that the beta bursts are related to brief and sudden release of mental tension.

\section{RESUME}

SURVENUE DE "BOUFHES BETA" AU NIVEAU DU ( ORTEX FRONTAI. CHEZ I.'HOMME, EN LIAISON AVEC CERTAINS PARAMETRES PSYCHOLOGIQUES

1. Chez un malade psychiatrique porteur d’électrodes implantées au niveau des diverses structures cérébrales. les auteurs ont observé la survenue d'une activité paroxystique de 16 à 26 $\mathrm{c} / \mathrm{sec}$ au niveau du cortex frontal inférieur.

2. Ces bouffées bêta surviennent au cours de situations de comportement "libre" mais peuvent être également provoquées par divers paradigmes stimulus-performance tel que le paradigme de VCN, le paradigme de choix binaire, etc.

3. Aucune relation significative n'a été relevée entre la survenue des bouffées bêta et une forme particulière de la VCN.

4. Une augmentation significative des bouffées bêta s'observe au cours de situations impliquant une prédiction de la part du malade.

5. Une diminution faible mais significative de la vitesse cardiaque s'observe lors de la survenue des bouffées bêta.

6. Les auteurs font l'hypothèse que les bouffées bêta seraient liées à un relâchement bref el soudain de la tension mentale.

We most gratefully acknowledge the most important support we have received from Dr. W. Grey Walter and his collaborators in the Burden Neurological Institute (Bristol) of whom particularly Dr. H. J. Crow has given invaluablo advice in the evaluation of the patient, the methodology and the therapy. The neurosurgical intervention and the postoperative care of the patient were performed by Dr. C.W. M. van Veclen, aided mainly by Miss J. G. W. Hooning van Duyvenbode.

\section{REFERENCES}

Crow. H. J.. Cooper, R. and Phitlips. D. G. Controlled multitocal frontal leucotomy for psychiatric illness. $J$. Newol. Neurosurg. Psychiat., 1961, 24: 353-360.

Deimas, A. et Pertuist1, B. Topométrie cranio-encéphalique che I homme. Cranio-cerebral topometry inman. Masson. Paris. Thomas. Springfield. Ill., 1959.

Green. J. D. and ARdini, A. Hippocampal electrical activities in arousal. $J$. Neurophlsiol. 1954, $17: 533-537$.

LACEY. J. 1. and LACEY, B. C. Experimental association and dissociation of phasic bradycardia and vertex negative waves: a psychophysiological study of attention and response-intention. Abstracts and int. Congr. on the contingent negatice rariation. Vancouver, B.C.. Canada. June. 1971

STORM VA: LEEUWEN. W. and KaMP, A. Radiotelemetry of EEG and other biological variables in man and dog. Proc. roy. Soc. Med., 1969, 62: 451-453

Storm van LeeuWen, W., Kamp, A., KoK, M. L. et Tilitin, A. M. Relation entre l'activité électrique de certaines structures cérébrales et le comportement du chien. Ret. newol.. 1963, 109: 258-259.

Storm vax Leeumen, W., Kamp, A., Kok, M. L. and TIElEN. A. M. Relations between behaviour in dogs and electrical activities in various parts of the brain. Progr. Brain Res., 1968, 22: 181-194.

Walthr. W. G., Cooper, R., Aldridgil, V. G. and MC CAIIUM. W. C. The contingent negative variation: an electrocortical sign of sensory-motor association and expectancy in the human brain. Nature (Lond.) 1964, 203: $380-384$. 